\title{
Design of Multimedia On-Demand Client Based on Big Data and Computer Network Technology
}

\author{
Zhengling $\mathrm{He}$ \\ Information and Modern Educational Technology Center, Xi'an University, 710065
}

Keywords: Computer; Network Technology; Big Data; Multimedia On-Demand Client

\begin{abstract}
With the continuous development of network and information technology, especially under the promotion of big data and computer network technology, new Internet media technologies are constantly developing to varying degrees. Image, video, audio and so on gradually take an indispensable position in media resources under the increasingly developed network, which combines traditional graphic information and other information into media information that is generally transmitted on the network. Streaming technology is deeply accepted and loved by various industry sectors due to its various characteristics. Based on this technology, it is extremely urgent to develop a multimedia resource on-demand system with shared functions and flexible implementation of user program on-demand and multimedia resource storage.
\end{abstract}

\section{A Brief Introduction to the Concept and Working Principle of Multimedia On-Demand System}

\section{Basic Concept of Multimedia on-Demand System}

The multimedia on-demand system is a new application field developed in the Internet in recent years. The system can not only send information to individual customers, but also simultaneously transmit information to multiple users. Compared with traditional TV systems that transmit data individually and are not actively received by users, the multimedia on-demand system not only realizes the user's autonomy to the greatest extent, but also realizes two-way video information transmission. The most important is that it allows users to actively choose to play content based on individual needs.

Nowadays, digital technology has completed the conversion of text images, video and audio signals, and the prior art has been able to complete the storage, transmission and display of such information. However, in the face of the existing user community's demand for video and audio fidelity quality, the existing technology can not fully meet the requirements, especially for the storage of large amounts of data and continuous playback requirements. With big data and computer network technology, we can try to make larger capacity storage devices, better bandwidth and network environment, and ensure real-time transmission of information in multimedia on-demand. The networked and stand-alone multimedia on-demand system is generally composed as shown below.

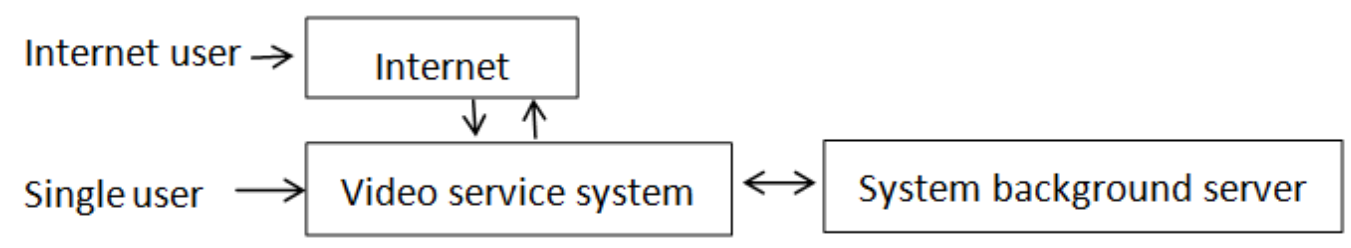

Figure1 Basic multimedia on-demand system

\section{Basic Working Principle of Multimedia On-Demand System}

High-definition, real-time multimedia on-demand systems typically consist of three main components, that is, users, client software and set-top box hardware. This system is designed to address the bandwidth services required by different user groups and networks of different sizes, 
and is structured by the Browser/Application Server/Database Server model. Through the design of the real-time data stream protocol and the control of the multimedia data transmission to enhance the adaptability of the system in different network environments. Thus, an advanced and efficient solution is built for the requirements of large data storage and real-time transmission. The overall structure and module division of the multimedia on-demand system based on streaming media is mainly as shown in the following figure.

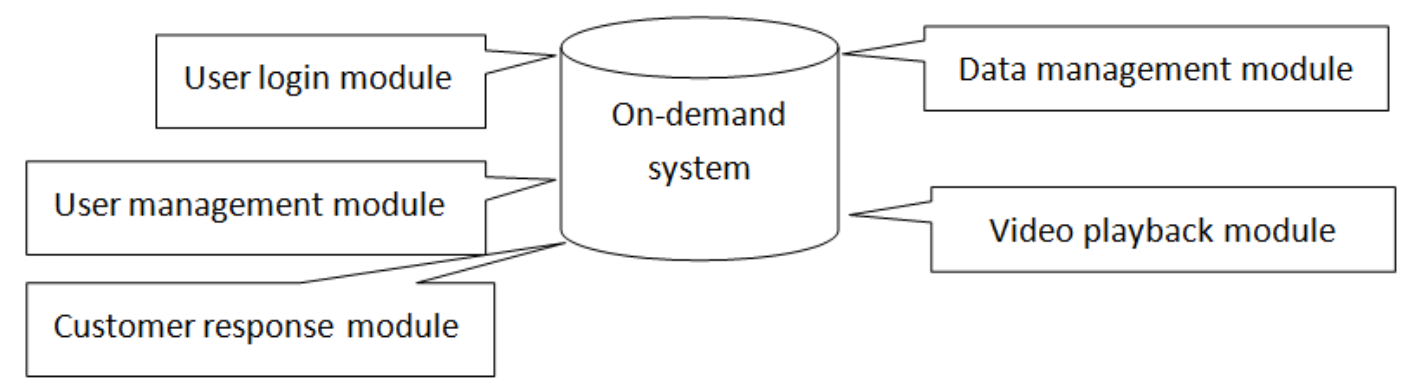

Figure2 multimedia on-demand system on streaming media

\section{Development Status of Multimedia On-Demand Client under Big Data and Computer Network Technology}

Nowadays, in the process of developing real-time multimedia communication services, storage technology, network and compression technology have become the pillars of the promoters, and streaming media technology, especially IP-based streaming media technology, is getting better and better. Nowadays, the use of streaming media, including video on-demand, has also developed rapidly. However, the quality of service of these applications cannot satisfy users. This is because the service method based on the server or the client is likely to cause the server performance to be unable to meet the demand. The server may be difficult to send the stream media to the user group with large traffic because of the limited I/O of the disk and the network. To solve the limitation of $\mathrm{C} / \mathrm{S}$ mode, it is first necessary to apply the $\mathrm{P} 2 \mathrm{P}$ peer-to-peer network technology to the video-on-demand system, then use the unstructured structure and select the Gossip type protocol for information retrieval. In other words, any node acting as a server will also receive services from other nodes while serving other nodes. Connections and data exchanges between nodes can minimize server load, in other words, better use of node resources. Under big data and computer network technology, the source of data is more broad, and both the type of data being played and the data of information are integrated into a broader scope. The application of big data and computer networks enables multimedia on-demand clients to seamlessly connect to TVs, mobile phones, tablets and more. As long as the user is connected to the network, they can search for the content they are looking for in the first batch of information. Big data-based data collection technologies, such as web crawlers, can be used for deep data mining from Internet, forums, Weibo and many other media. The mined information can be static, such as pictures, text, etc., or it can be dynamic, such as video, voice, and so on. In the context of big data, the excavated information can be uniformly arranged and processed by modern data processing technology, and different data types can be flexibly transformed, and finally enter the on-demand system in a user-selected manner.

Nowadays, media playback technology is increasingly moving towards intelligent direction. The basis of all this is the analysis and processing of data. Big data technology provides a broad space for the intelligent development of media playback. The keywords and themes of information and data under big data technology can be arranged and identified according to specific algorithms. Data analysis is strictly normative and it allows end users to have clear and intuitive access to different forms of information, which is more conducive to customer's on-demand selection. Through the in-depth analysis of big data, we can provide more and more personalized services to our customers and continuously promote the transformation of media technology. Multimedia 
on-demand technology based on big data and computer network technology is being combined with artificial intelligence and smart home in more and more places. At the same time, intelligent video on-demand technology is constantly being implanted in various mobile phone applications, enriching and facilitating our work and life in many fields.

\section{System Functions, Design Concepts and Technical Requirements of Current Multimedia On-Demand Client Design}

\section{System Function Requirements for Current Multimedia On-Demand Client Design}

The simplest and more traditional multimedia on-demand system is a video on demand system based on "file sharing method". The main feature of the system is that it does not require additional application software, which is fundamentally different from streaming video on demand systems. The design of the network protocol must focus on the reliability of the network and the timeliness of non-information transmission, so it is transmitted in burst mode. Multimedia applications need to transmit real-time audio and video information, and must be transmitted in a continuous "stream" manner with a transmission delay of milliseconds.

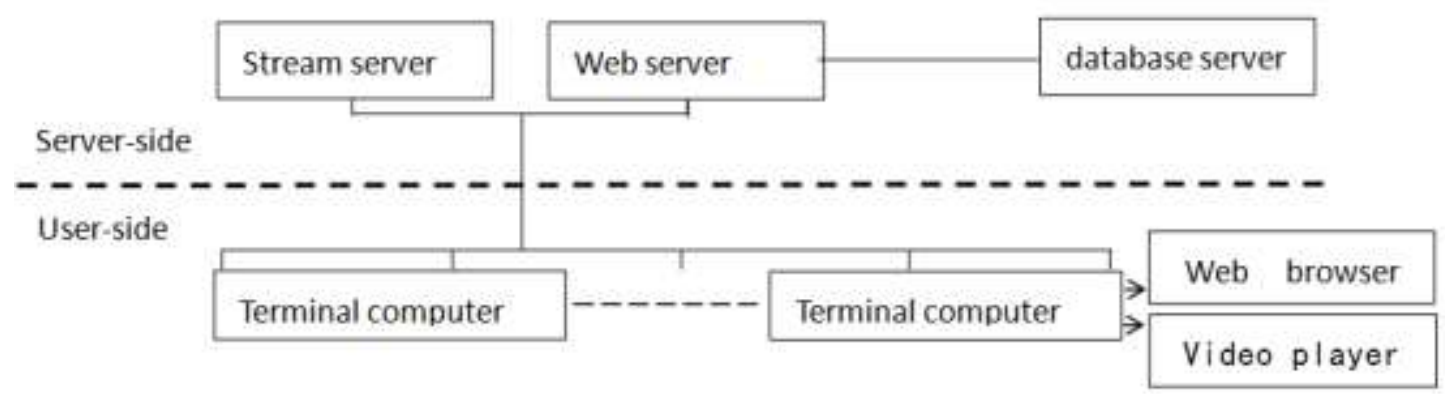

Figure 3 current multimedia On-demand client

As shown above, the components of a multimedia on demand system usually consist of two parts, that is, a video server and a client browser.

\section{Video Server}

The main role of the video server is to provide customers with a continuous and stable video data stream, and to directly play, fast forward, rewind and pause the video stream. The digitized video program is stored on the disk array of the video server. The amount of programs stored depends mainly on the configured hard disk size. For example, storing $100 \mathrm{~h}$ programs in MPEC-1 (1.5 $\mathrm{Mbps}$ ) format requires about $55 \mathrm{c}$ of hard disk space.

\section{Client B rowser}

The client browser is primarily responsible for communicating with the server and obtaining information about the program. It is located at the user terminal for the viewer to watch audio, video and other programs transmitted from the network. It has a certain degree of scalability in terms of scale. For example, when a large number of viewers watch the same program, the problem of network jam and audio quality is not easily caused. In addition, its user-friendly interface makes it easy to control and operate. The video window can be set to full screen by the user without additional bandwidth.

General Concept Requirements for Multimedia On-Demand System Design under Big Data and Computer Network Technology

Under the premise of big data and computer network technology, the design of multimedia on-demand client must consider the following three most important points, that is, core function, user experience and function introduction.

\section{Core Function}

The core function is to clarify the actual needs provided by the on-demand client, and to highlight its professionalism and diversification in design, so that it can let users understand easily 
and try its best to respond accurately and quickly to user needs.

\section{User Experience}

User experience is a major part of the interest of all Internet service industries. Any product or service platform must put the user experience first, which requires the use of big data technology to complete. First of all, the platform needs to analyze the service objects of the on-demand system, including their age, gender, hobbies, spending power and other factors. Then, when designing the page, vote for it and recommend it to the user in the context of big data. In addition, the platform also needs to do certain precautions in the auditing system or in the comment area. It is strictly forbidden to display words such as illegal words and deeds or language attacks in front of users. This is also a preventive work for the user's experience environment.

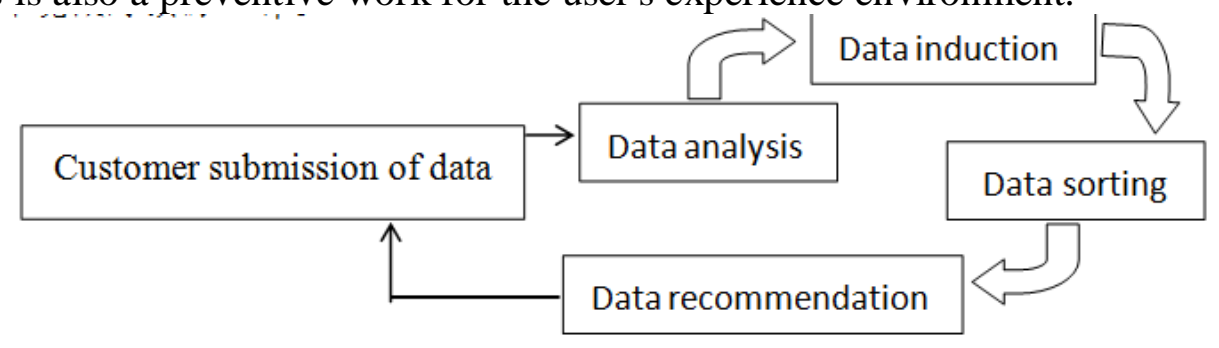

Figure4 User experience system

\section{Function Introduction}

The function introduction, as the name suggests, refers to the page layout of the client. The keyword matching of the column is also extremely important. Under the premise of understanding the actual needs of the client, the keyword of the column is the best way to guide the user to use quickly and conveniently. Secondly, the media player client should be simple and easy to understand, so that the viewer is not easy to have a sense of disgust and so on. Furthermore, the interface processing of commercial advertising of software is also important. Commercial advertising belongs to the platform benefits, and how to distribute these advertisements mainly depends on the analysis of big data. However, these advertisement pages cannot affect the normal use of the user in the platform, but also must be conspicuous, while maintaining the user experience, while maintaining the interests of the software platform.

Nowadays, big data has become the mainstream application in the Internet age, and it also brings more convenience to people's lives, saving a lot of time for people's work and lives.

\section{System Hard ware and Software Platform}

\section{Video On-Demand Server}

The core component of the hardware environment is the video on-demand server. The system installs Real Server 8 on this server, which function is equivalent to a TV signal transmitting station. In the case of a large number of viewers, higher requirements should be placed on the configuration of the server.

\section{Web Server}

The web server can be used for the release of the system website. The Microsoft Internet Information Server 6.0 system is installed on this server, and the website pages and applications will be installed on this server.

\section{Database Server}

This server is used to store video, audio and other data information. Microsoft SQL Server 2005 is installed on this server. The background data of the system website is stored on this server.

\section{Hard Disk Array}

Since the TV center video on-demand system has a huge amount of video information, it usually needs to store a very large number of files such as video and audio, so the configuration of the large-capacity storage device is indispensable.

\section{Server Operating System}

Install Microsoft Windows 2003 server. 


\section{Cloud Computing Server System}

Cloud computing is widely regarded as the third revolution in the IT industry since computer and network technology. Cloud computing has changed the way people get and process information. Cloud computing leverages the transmission capabilities of high-speed computer networks to move data processing to large cloud computing centers. The characteristics of a widely recognized cloud computing server can be summarized into four aspects, namely high density, low energy consumption, easy management and system optimization. The close integration of cloud computing and computer technology under big data has successfully realized the resource sharing and information exchange between computers and other devices, so that users can browse various video information without having to save various types of data locally.

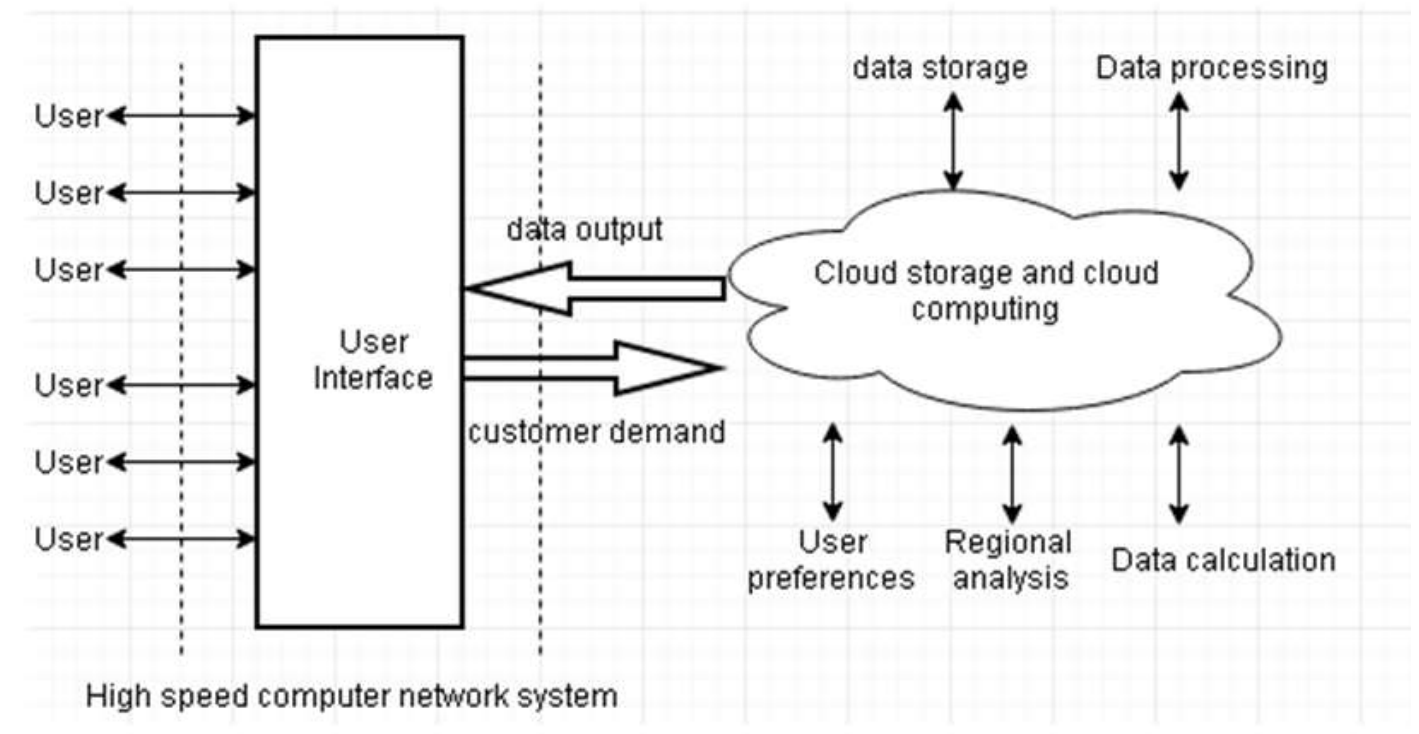

Figure5 Cloud computing system

\section{Conclusion}

Today, computers and cloud computing technologies are simultaneously developing higher levels of technology. At the same time, big data technologies have been able to handle huge, irregular and non-data content and information. Unprecedented changes in computer networks, cloud data processing, artificial intelligence, and many other areas have become the most important information of the times. In this context, the application and development of multimedia on-demand systems have also been promoted. The client design of the system is based on the new concept of "user autonomy", which realizes the ideal of playing video and audio programs according to users' needs. Through the system, thousands of customers can broadcast multimedia information of images, pictures, texts and voices they need, which provides a more convenient and flexible experience for users.

\section{Reference}

[1] L. Chen. Thoughts on Campus Culture Construction under the New Network Environment of Computer Network [J]. Journal of Liaoning Higher Vocational College, 2014 (3): 100-102.

[2] P. Dong. Discussion on the New Media Development Mode Based on Computer Network Technology [J]. Information Security and Technology, 2015 (9): 15-16.

[3] X. H. Qin. Talking about the Influence of Computer Network Technology Development on Internet New Media [J]. Computer CD-ROM Software and Application, 2013 (23): 33-34.

[4] W. X. Li, Y. H. Lin and C. Weng. Application and Research of Computer Network under the New Media Paradox [J]. China Media Technology, 2013 (22): 73.

[5] Y. J. Liu and Y. Peng. Application and Research of Computer Network in New Media 
Environment--A Review of Computer Network Application Technology Tutorial (Fourth Edition) [J]. Young Reporter, 2016 (32): 104.

[6] Q. X. Zeng and X. D. Shi. Analysis of New Media Development Based on Computer Network Technology [J]. Science \& Technology Innovation and Application, 2016 (26): 91.

[7] Y. F. Jin. Traditional Media Is Involved in News and Information APP [N]. China News and Publications, 2013.5. 